\title{
ImageCompression Using Real Fourier Transform, Its Wavelet Transform And Hybrid Wavelet With DCT
}

\author{
Dr. H.B.Kekre \\ Sr. Professor, MPSTME, \\ Department of Computer \\ Engineering \\ NMIMS University \\ Mumbai, India
}

\author{
Dr. Tanuja Sarode \\ Associate Professor \\ Department of Computer \\ Engineering, TSEC \\ Mumbai University \\ India
}

\author{
Prachi Natu \\ $\mathrm{Ph}$. D. Research Scholar \\ MPSTME, NMIMS University \\ Mumbai, India
}

\begin{abstract}
This paper proposes new image compression technique that uses Real Fourier Transform. Discrete Fourier Transform (DFT) contains complex exponentials. It contains both cosine and sine functions. It gives complex values in the output of Fourier Transform. To avoid these complex values in the output, complex terms in Fourier Transform are eliminated. This can be done by using coefficients of Discrete Cosine Transform (DCT) and Discrete Sine Transform (DST). DCT as well as DST are orthogonal even after sampling and both are equivalent to FFT of data sequence of twice the length. DCT uses real and even functions and DST uses real and odd functions which are equivalent to imaginary part in Fourier Transform. Since coefficients of both DCT and DST contain only real values, Fourier Transform obtained using DCT and DST coefficients also contain only real values. This transform called Real Fourier Transform is applied on colour images. RMSE values are computed for column, Row and Full Real Fourier Transform. Wavelet transform of size $\mathrm{N} 2 \times \mathrm{N} 2$ is generated using $\mathrm{NxN}$ Real Fourier Transform. Also Hybrid Wavelet Transform is generated by combining Real Fourier transform with Discrete Cosine Transform. Performance of these three transforms is compared using RMSE as a performance measure. It has been observed that full hybrid wavelet transform obtained by combining Real Fourier Transform and DCT gives best performance of all. It is compared with DCT Full Wavelet Transform. It beats the performance of Full DCT Wavelet transform. Reconstructed image quality obtained in Real Fourier-DCT Full Hybrid Wavelet Transform is superior to one obtained in DCT, DCT Wavelet and DCT Hybrid Wavelet Transform.
\end{abstract}

\section{Keywords-Real Fourier Transform; Hybrid Wavelet Transform; DCT}

\section{INTRODUCTION}

Image compression is storing images using lesser number of bits than its original size. Image compression leads to less storage space and less bandwidth for transmission. Hence in this world of internet and multimedia applications image compression is of utmost important and interesting area to work on. It is used to store images in medical image database, to generate image database in biometrics and many other applications. Image compression is divided into two categories: lossy and lossless [1]. Compression ratio and image quality of decompressed image, these are two major things to be considered in image compression. As compression ratio increases, quality of reconstructed image starts degrading. Many compression techniques like vector quantization, predictive coding, differential image coding, transform coding have been introduced. Transform based techniques are popular for image compression especially at low bit rate. In transform domain, many researchers have worked on image compression and still this area is equally popular to work on. Discrete Cosine Transform is widely used. It separates an image into different frequency components. Low frequency components are located at top left corner giving high energy compaction. High frequencies are located in bottom right corner. Elimination of these high frequency elements gives transformed image with few low frequency components. If image is reconstructed from such lesser number of transformed, low frequency elements, it gives compressed image without losing much data contents in original image. Wavelet transform coding is preferred over simple orthogonal transform in image compression due to its multi-resolution property. It provides enhanced image quality even at higher compression ratios [2]. Recently hybrid transformation techniques have come into picture which combines properties of two different transforms [3]. It gives compressed image with visually perceptible image quality. This paper focuses on Real Fourier Transform which is an orthogonal transform, obtained by combining cosine and sine coefficients of Fourier transform. Wavelet transform is generated from orthogonal Real Fourier Transform by using the algorithm in [4] and also hybrid wavelet is generated by following the procedure in [5] and compression of image is studied using all these three transforms. Remaining paper is organised as follows: Section II contains brief introduction to related work. Section III discusses proposed technique. In proposed technique, orthogonal Real Fourier Transform, Real Fourier Wavelet Transform and Real Fourier Hybrid Wavelet Transform are discussed. In section IV results obtained from experimental work are discussed and finally conclusion is drawn in section $\mathrm{V}$.

\section{RELATED WORK}

A lot of work has been done on image compression and still it is going on. Image compression using biorthogonal wavelet transform is proposed by liu in [6]. A lifting scheme wavelet based transform with a modified entropy coding algorithm is proposed in [7]. It discusses effect of block sub-band coding on compression factor and quality of an image. Hybrid DCT-VQ approach is presented in [8] where high frequency elements are first removed by applying 2D-DCT on colour image and then VQ algorithm is applied on this reduced $2 \mathrm{D}$ vector to speed up the process. Use of wavelet transform started with Haar 
transform. In [9] Adman Khashman et.al. Proposed an image compression technique using neural network and Haar wavelet transform. Their paper used Haar wavelet compression with nine compression ratios and a supervised neural network that learns to correlate the gray image intensity with a single optimum compression ratio. Two neural networks getting different input image sizes are implemented in this technique and a comparison between their performances was presented. A combined approach of image compression based on vector quantization and wavelet transform is proposed using RBF neural network in [10] which works on grayscale images.

\section{PROPOSED TECHNIQUE}

This paper proposes a new transform called real Fourier transform. We know that Fourier transform is actually implemented using complex numbers, where the real part is weight of cosine and imaginary part is weight of sine. DCT is similar to Fourier Transform but uses only cosine functions i.e. using only real and even functions. Discrete Sine Transform (DST) uses only sine functions i.e. real and odd functions. DCT and DST contain only real data sequence. Both DCT and DST can be computed by using two FFT's of original data sequence of length $\mathrm{N}$. i.e. they are equivalent to FFT of twice the length[11]. To avoid complex values in the output like Discrete Fourier transform, combination of DCT and DST coefficients are considered to form transformation matrix. It gives Real Fourier Transform. Thus transform matrix contains only real values and no complex exponentials. Using this transform matrix column transform, row transform and full transform of image is obtained [12]. In column transformed image, energy compaction occurs towards upper portion of the transformed image. In row transformed image energy compaction takes place towards left portion of an image whereas in full transformed image high energy coefficients are present at top left corner. After applying transform, low energy coefficients are eliminated to represent the image in lesser number of bits. Hence in column transform we eliminate rows in lower portion and in row transform, columns at the right side of transformed image are eliminated. In full transform low energy coefficients in both, rows and columns are eliminated. Same numbers of coefficients are eliminated in above three cases and results are compared. Steps to apply transform on an image are as follows:

\section{A. Column, Row and Full Transform}

- Separate R, G, and B plane of an image.

- Generate Real Fourier Transform matrix ' $\mathrm{T}$ ' of size

- $\quad 256 x 256$, since image size is $256 \times 256$.

- Apply transform on each plane of image separately.

- For column transform use $\mathrm{F}=[\mathrm{T}] *[\mathrm{f}]$, where $\mathrm{f}$ is original image and $\mathrm{F}$ is transformed image.

For row transform use $\mathrm{F}=[\mathrm{f}]^{*}[\mathrm{~T}]^{\mathrm{T}}$ where

$[\mathrm{T}]^{\mathrm{T}}$ is transpose of transform matrix.

For full transform use $\mathrm{F}=[\mathrm{T}]^{*}[\mathrm{f}]^{*}[\mathrm{~T}]^{\mathrm{T}}$
- From column/row/full transformed image eliminate specific number of low energy coefficients. It is done by eliminating columns/rows/combination of columns and rows respectively. Apply inverse transform to reconstruct the image from compressed image.

- Calculate Root mean Square Error between original image and reconstructed image.

- Compare RMSE values in column/ row/ full transform to compare their performance

\section{B. Real Fourier Wavelet Transform}

Use of wavelets is beneficial over simple orthogonal transform because it concentrates on local characteristics of image. Using algorithm in [5], $\mathrm{N}^{2} \mathrm{xN}^{2}$ Real Fourier Wavelet Transform matrix is generated from $\mathrm{NxN}$ Real Fourier Transform matrix. Using the steps 1 to 8 in case (A) of proposed technique, column, Row and full Real Fourier Wavelet Transform of image is obtained and performance of each is compared.

\section{Real Fourier Hybrid Wavelet Transform}

Orthogonal transforms are used to analyse global properties. Each transform has its own characteristics. In wavelet transform, some orthogonal transform focuses on global properties of data whereas some exhibit local properties in better manner. Hybrid wavelet combines properties of two different orthogonal transforms giving strengths of both transforms. Here two different transforms selected are: Real Fourier Transform and Discrete Cosine Transform. Following the methodology in [6], Real Fourier-Cosine Hybrid wavelet Transform matrix is generated. $8 \times 8$ Real Fourier transform matrix and $32 \times 32$ size Cosine matrix is used to generate 256x256 size Real Fourier-Cosine Hybrid wavelet Transform matrix. Column, Row and Full Hybrid wavelet Transform of images is computed using steps 1 to 4 in section (A). In hybrid wavelet transform, energy of each row/ column is calculated and rows/columns are sorted in descending order of their energy. Rows/columns having lowest energy are then eliminated to compress the image, i.e. now steps 5 to 8 in section (A) are used to compute RMSE. Results in each case are computed using RMSE as a measuring parameter. Also, the performance of DCT column transform[12], DCT column wavelet transform[13] with different sizes of two Cosine Transform matrices, Real Fourier Column transform, Real Fourier column wavelet transform and Real Fourier-Cosine column hybrid wavelet transform is compared.

Similarly, DCT row transform, DCT row wavelet transform with different matrix sizes, Real Fourier row transform, Real Fourier row wavelet transform and Real Fourier-Cosine row hybrid wavelet transform are compared.

All these six cases are considered for full transform and compared. 

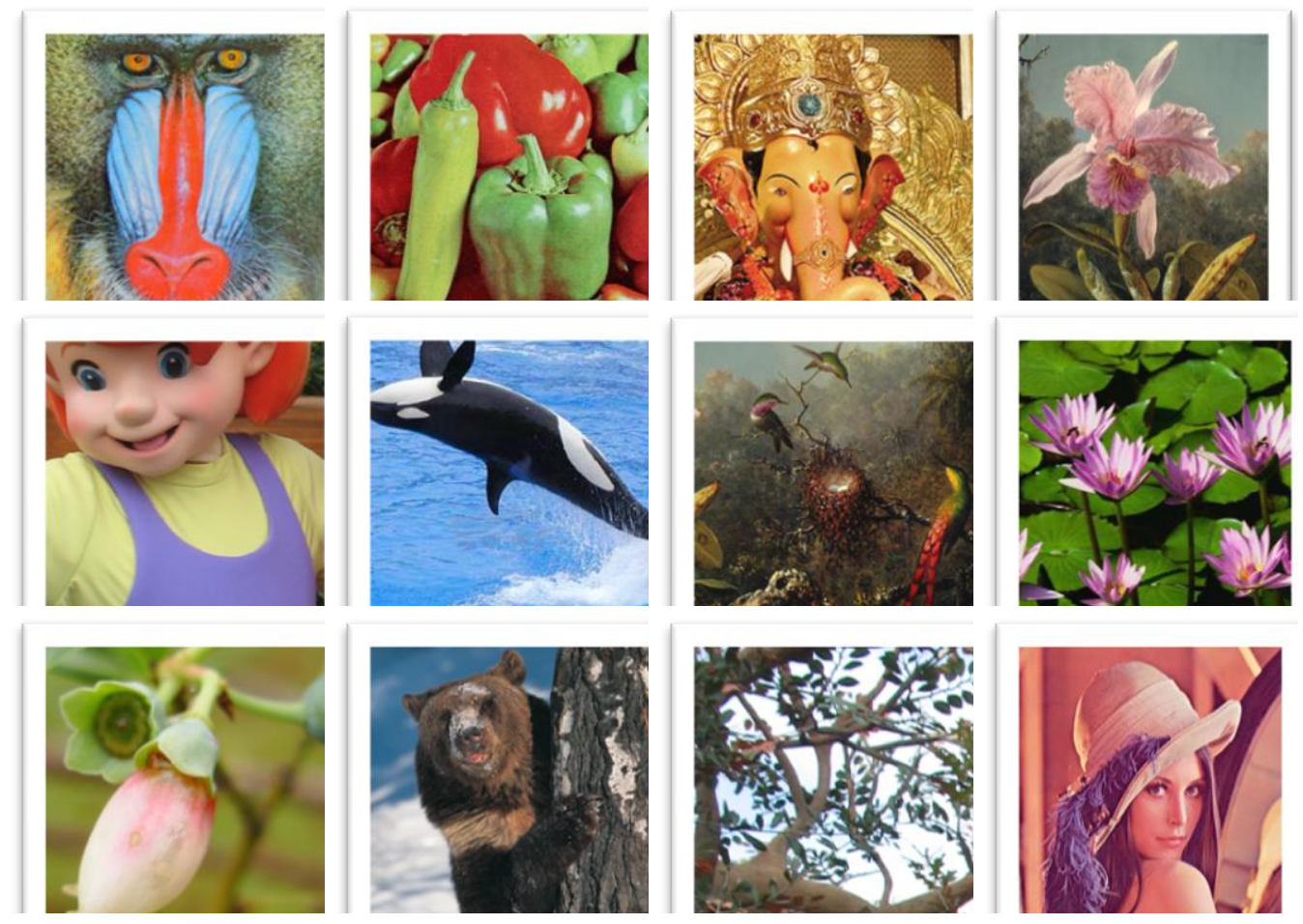

Fig.1. Set of twelve test images of different classes used for experimental purpose namely (from left to right and top to bottom) Mandrill, Peppers, Lord Ganesha, Flower, Cartoon, dolphin, Birds, Waterlili, Bud, Bear, Leaves and Lenna

\section{Results AND Discussions}

Proposed technique is applied on set of colour images. Twelve different colour images are used for experimental purpose. Each image is of size 256x256. Matlab 7.2 on AMD dual core processor is used for experimentation. Fig.1 shows sample colour images used for experimental purpose.

Graph in Fig.2 compares the results of Column, Row and Full Real Fourier Transform. It has been observed that approximately same results are obtained for Column and Row transform. When more numbers of coefficients are eliminated i.e. higher compression ratio, there is considerable difference between RMSE values of Full transform, column transform and row transforms. Elimination of 240 rows from 256 rows of transformed image gives compression ratio 16. At this compression ratio, RMSE value is19.734 in Full transform. In column and row transform it is nearly 25 .

Fig. 3 shows graph of RMSE values for Real Fourier Wavelet transform with three different cases: Column wavelet, Row wavelet and Full wavelet transform. Here 256x256 size transform matrix is obtained from $16 \times 16$ size Real Fourier Transform matrix.

From figure, it can be observed that in Real Fourier Full Wavelet Transform, at higher compression ratio, RMSE values are decreased by more than half as compared to column wavelet and row wavelet transform. It means image quality of reconstructed image obtained by Real Fourier Full Wavelet Transform is much better than obtained in Column Wavelet and Row Wavelet Transform.

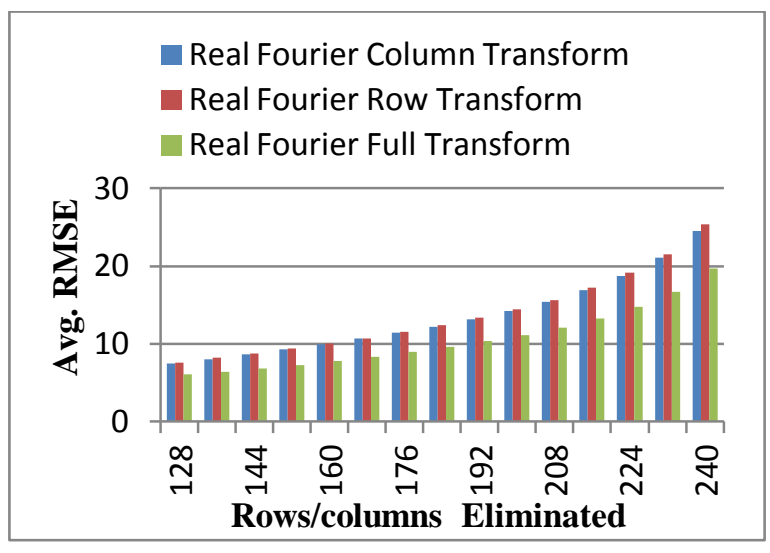

Fig.2. Comparison of RMSE in Real Fourier Column Transform, Real Fourier row Transform and Real Fourier Full Transform

Fig. 4 compares the performance of Real Fourier Hybrid Wavelet Transform. Here two different transforms are used to generate hybrid transform matrix. Hybrid Transform matrix size is same as that of image size. First, Real Fourier Transform of $8 \times 8$ is considered and second Discrete Cosine Transform of $32 \times 32$ is selected $256 \times 256$ Hybrid Transform matrix is generated using algorithm in [6]. Computed RMSE values for Column, Row and Full Real Fourier-Cosine Hybrid Transform are compared in following figure. This graph clearly indicates that, RMSE values are reduced to one third at higher compression ratios when Full Hybrid wavelet Transform is applied on an image. It assures better image quality even when more coefficients are removed from transformed image. 


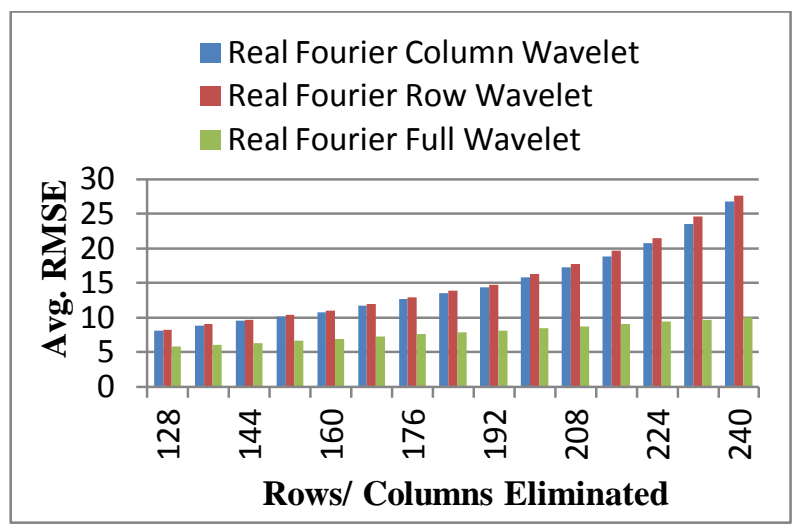

Fig.3. Comparison of RMSE in Real Fourier Column Wavelet Transform, Real Fourier Row Wavelet Transform and Real Fourier Full Wavelet Transform

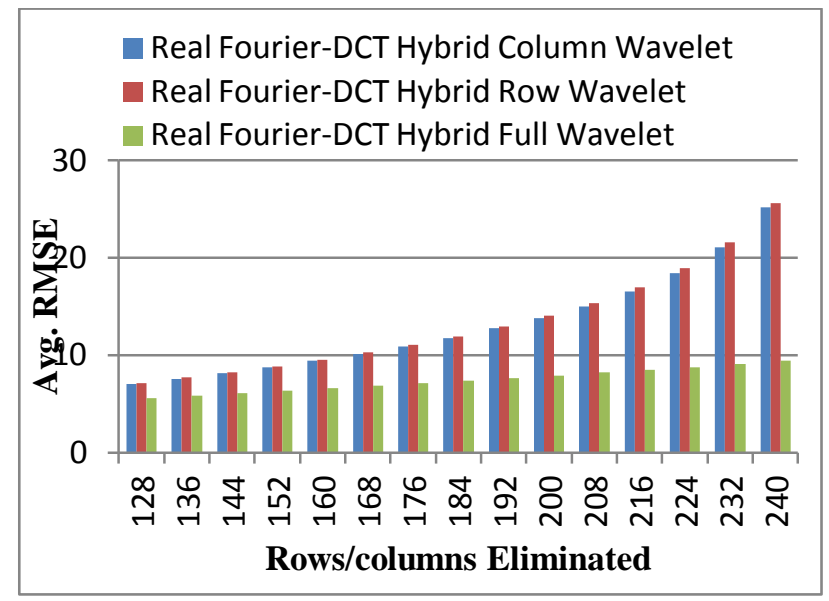

Fig.4. Comparison of RMSE in Real Fourier Hybrid Column Wavelet Transform, Real Fourier Hybrid Row Wavelet Transform and Real Fourier Hybrid Full Wavelet Transform

Fig. 5 compares RMSE values for column transforms of DCT, Real Fourier Transform and their combination. When we keep on removing more number of rows, DCT column wavelet transform (with $\mathrm{m}=8, \mathrm{n}=32$ ) and Real Fourier-DCT column Hybrid Wavelet Transform gives same results. It is because Cosine matrix of $32 \times 32$ is used in both cases to extract local properties of image. These results are slightly better as compared to DCT column, DCT column wavelet, Real Fourier column and Real Fourier column wavelet transform. Elimination of 240 rows from 256 rows gives compression ratio 16. At this high compression ratio DCT column Transform gives slight better performance.
In Fig. 6, performance for different cases of row transform is shown. It is nearly same as different cases of column transform. As we eliminate more number of columns, RMSE increases. Comparison of Full Transforms of Cosine, Real Fourier and their combination is given in Fig.7. From figure; it has been observed that Full Hybrid Wavelet Transform gives better performance than orthogonal Full Wavelet Transform. Among DCT Full and Real Fourier-DCT Full Hybrid Transform, second combination gives better results.

Fig. 7 shows results of Real Fourier- DCT Full Wavelet Transform. It gives the best results among all. Even after eliminating 240 rows, RMSE value of 9.4 is obtained. It indicates that perceptible image quality is obtained using this transform by retaining small amount of data.

Fig. 8 shows reconstructed images using different six cases of Column transform mentioned in Fig. 5. Well known 'pepper' image is selected to demonstrate the quality of reconstructed image. Fig. 9 shows reconstructed images for different Row transforms. For Full transforms, reconstructed images are shown in fig. 10. Comparison of Fig. 8,9,10 clearly indicates that Real Fourier-DCT Full hybrid Wavelet Transform gives best quality of reconstructed image among all.

\section{CONCLUSION}

In this paper Real Fourier Transform is studied. It is applied on the image in three ways: Column transform, Row transform and Full transform. Performance of these three is compared and it is found that Full transform gives better results than row and column Real Fourier transform. From NxN Real Fourier transform wavelet of $\mathrm{N}^{2} \mathrm{xN}^{2}$ is generated. In this case also Real Fourier Full Wavelet transform gives better compression than Column and Row wavelet Transform. Among Real Fourier Full wavelet Transform and Real Fourier Full Transform, Full Wavelet transform gives much better results when more number of coefficients are eliminated to get high compression ratio.

Also Real Fourier-DCT Hybrid Wavelet Transform matrix is generated and results for column, row and full hybrid transform are considered. Full hybrid wavelet transform gives better results than full transform as well as full wavelet transform. All results of Real Fourier Transform are also compared with and results of Cosine Transform, Cosine Wavelet transform and Cosine-Cosine Hybrid Wavelet Transform. It has been observed that, Real Fourier-Cosine Full Hybrid Wavelet Transform outperforms all other transforms giving lowest value of RMSE 9.407 when 240 rows from 256 rows of transformed image are eliminated. It gives perceptible image quality at compression ratio 16. 


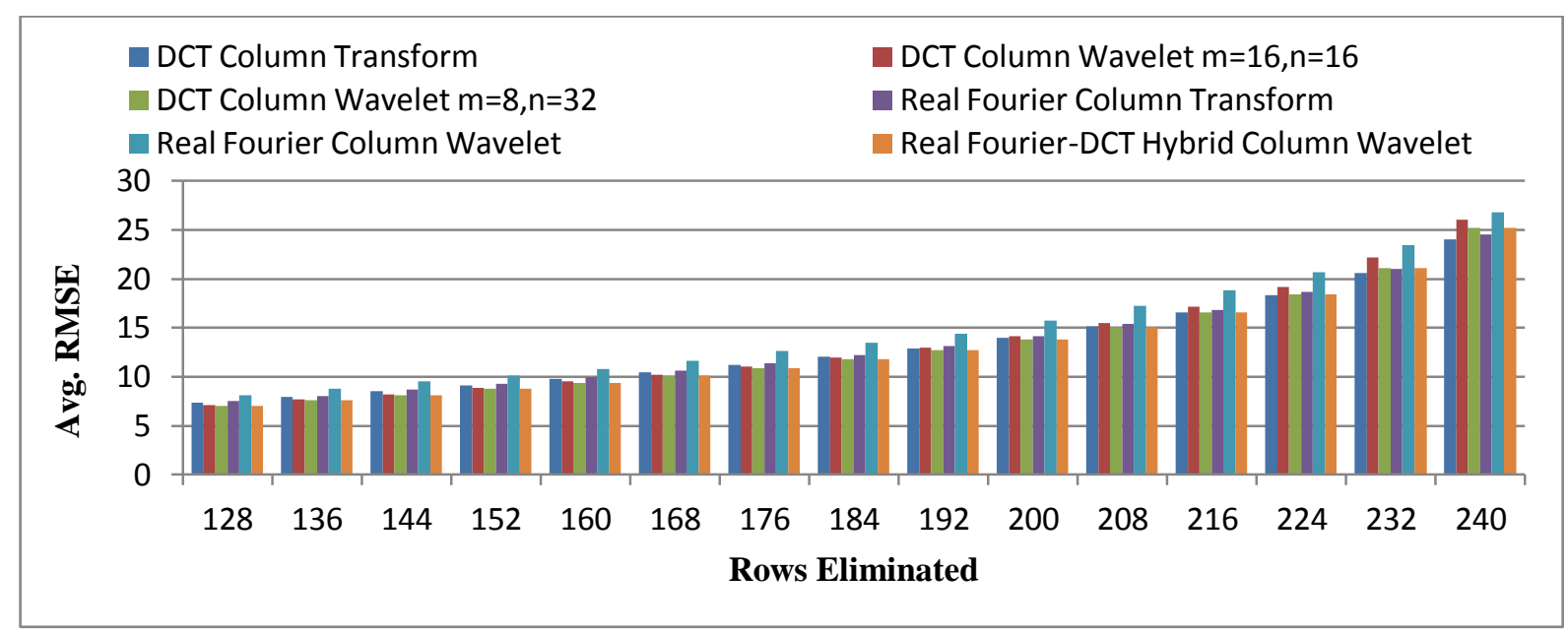

Fig.5. Comparison of RMSE for different cases of Column Transforms of DCT and Real Fourier Transform: DCT Column Transform, DCT Column Wavelet Transform $(m=16, n=16)$, DCT Column Wavelet Transform $(m=8, n=32)$, Real Fourier Column Transform, Real Fourier Column Wavelet Transform Real Fourier-DCT Hybrid Column Wavelet Transform $(m=8, n=32)$.

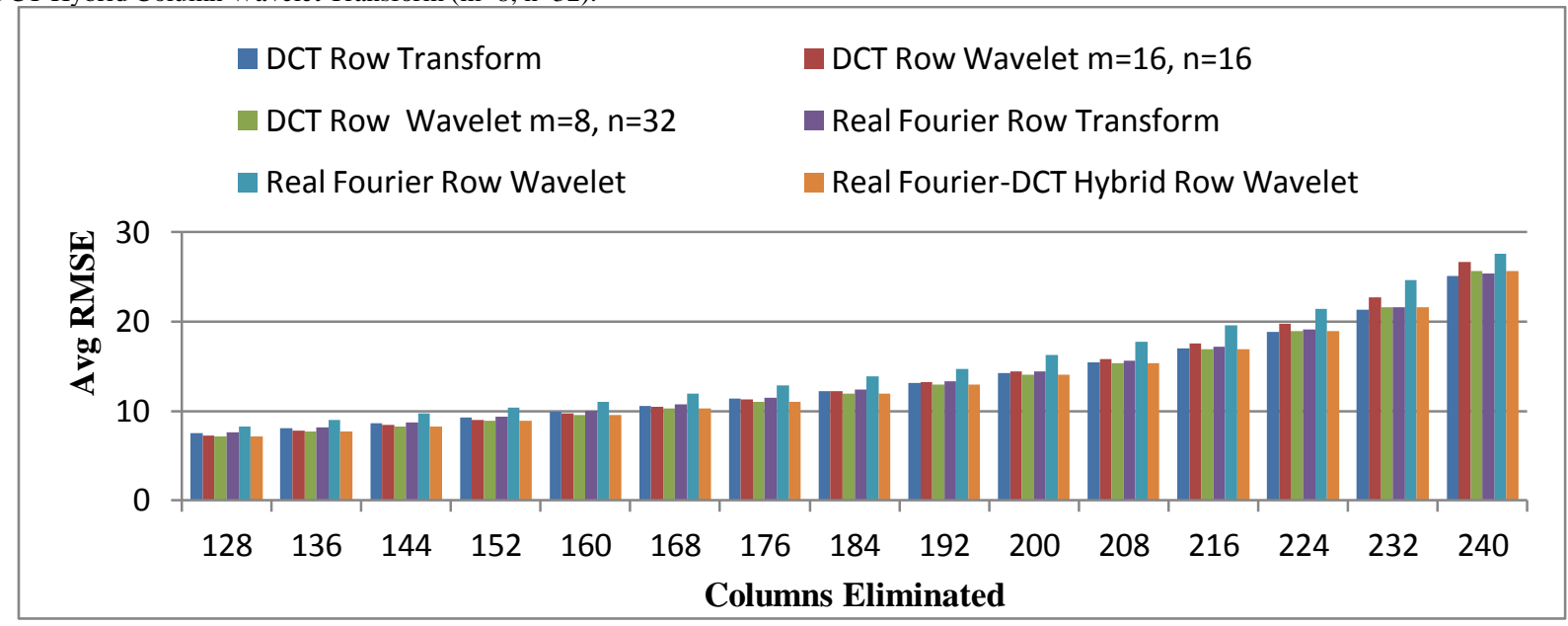

Fig.6. Comparison of RMSE for different cases of Row Transforms of DCT and Real Fourier Transform: DCT Row Transform, DCT Row Wavelet Transform $(m=16, n=16)$, DCT Row Wavelet Transform $(m=8, n=32)$, Real Fourier Row Transform, Real Fourier Row Wavelet Transform Real Fourier-DCT Hybrid Row Wavelet Transform

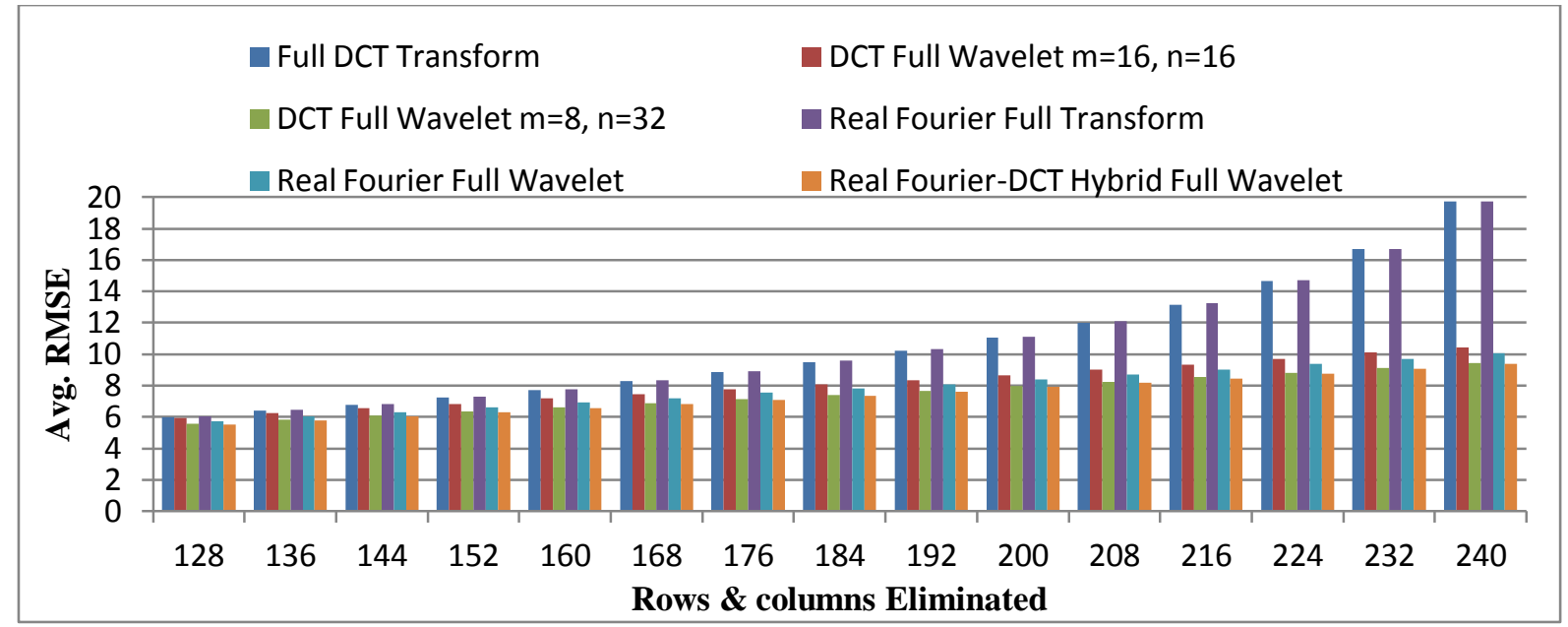

Fig.7. Comparison of RMSE for different cases of Full Transforms of DCT and Real Fourier Transform: DCT Full Transform, DCT Full Wavelet Transform $(\mathrm{m}=16, \mathrm{n}=16)$, DCT Full Wavelet Transform $(\mathrm{m}=8, \mathrm{n}=32)$, Real Fourier Full Transform, Real Fourier Full Wavelet Transform, Real Fourier-DCT Hybrid Full Wavelet Transform 

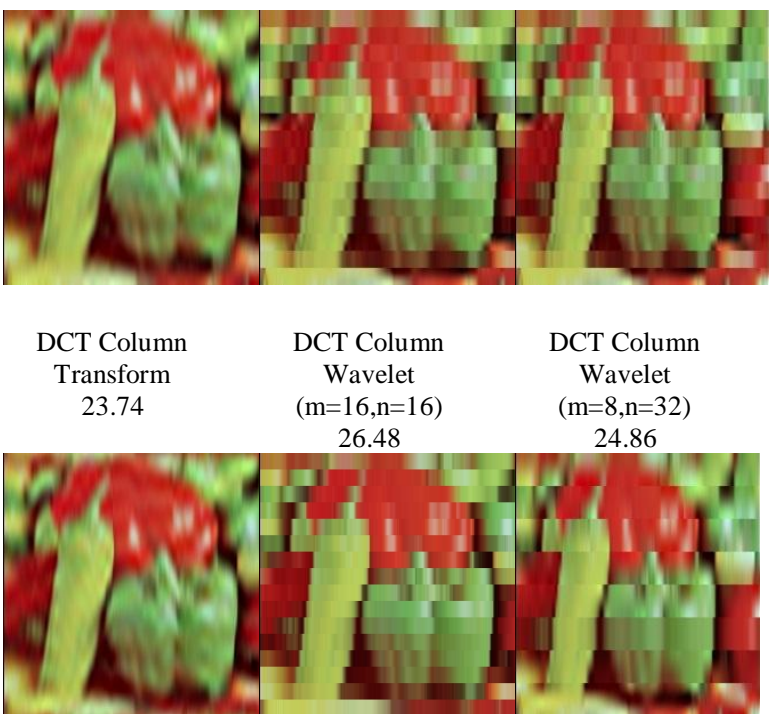

$\begin{array}{ccc}\begin{array}{c}\text { Real Fourier } \\ \text { Column Transform }\end{array} & \begin{array}{c}\text { Real Fourier } \\ \text { Column Wavelet } \\ (\mathrm{m}=16, \mathrm{n}=16)\end{array} & \begin{array}{c}\text { Real Fourier-DCT } \\ \text { Column Hybrid }\end{array} \\ \begin{array}{cc}\text { Wavelet } \\ 23.93\end{array} & 26.83 & (\mathrm{~m}=8, \mathrm{n}=32) \\ & & 24.86\end{array}$

Fig.8. Reconstructed images with RMSE values using six different cases of column transform when 240 rows of $256 \times 256$ images are eliminated

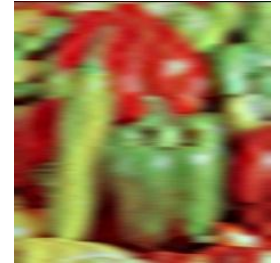

DCT Row Transform 25.80

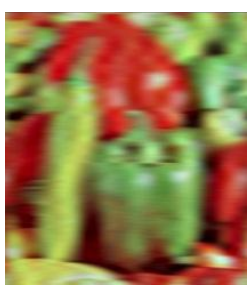

Real Fourier Row

Transform

25.83

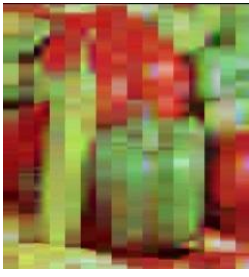

DCT Row Wavelet $(\mathrm{m}=16, \mathrm{n}=16)$ 27.68

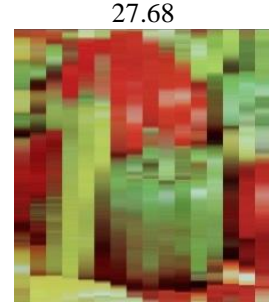

Real Fourier Row Wavelet $(m=16, n=16)$ 28.36

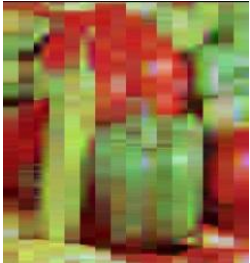

DCT Row Wavelet $(\mathrm{m}=8, \mathrm{n}=32)$ 26.78

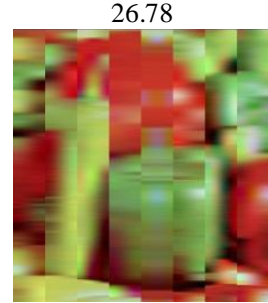

Real Fourier-DCT Row Hybrid Wavelet $(\mathrm{m}=8, \mathrm{n}=32)$ 26.77
Fig.9. Reconstructed images with RMSE values using six different cases of Row transform when 240 columns of 256x256 images are eliminated

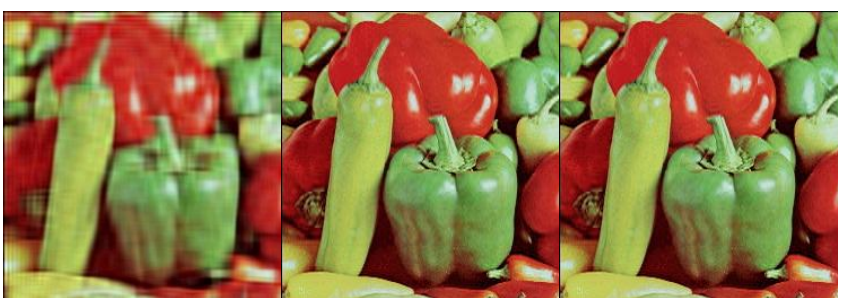

\section{DCT Full Transform 17.69}

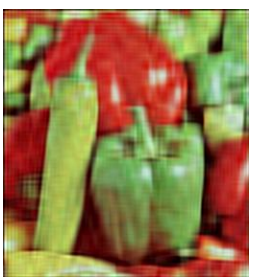

Real Fourier Full Transform 18.20
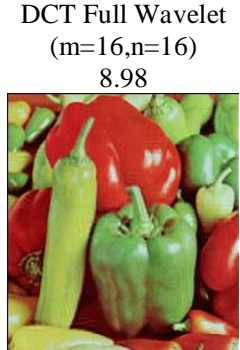
Real Fourier Full
Wavelet $(\mathrm{m}=16, \mathrm{n}=16)$

9.33

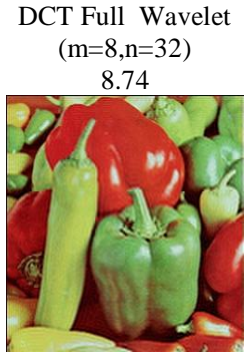

Real Fourier-DCT Full Hybrid Wavelet $(\mathrm{m}=8, \mathrm{n}=32)$ 8.641
Fig.10. Reconstructed images with RMSE values using six different cases of full transform when 240 rows/columns of 256x256 images are eliminated

\section{REFERENCES}

[1] Prabhakar.Telagarapu, V.Jagan Naveen, A.Lakshmi Prasanthi, G.Vijaya Santhi, "Image Compression using DCT and wavelet ansformation", IJSPIPPR, vol 4, issue 3, pp. 61-74, Sept 2011.

[2] M. J. Nadenau, J. Reichel, and M. Kunt, "Wavelet Based Color Image Compression: Exploiting the Contrast Sensitivity Function," IEEE Transactions Image Processing, Vol.12, no.1, pp. 58-70, 2003.

[3] Rachana Dhannawat, Tanuja Sarode, H. B. Kekre, "Kekre's Hybrid Wavelet TransformTechnique with DCT, Walsh, Hartley and Kekre's Transform for Image Fusion", International Journal of Computer Engineering and Technology(IJCET), Vol. 4, Issue 1, Feb 2013, pp. 195202.

[4] H.B.Kekre, Tanuja Sarode, sudeep Thepade, Sonal Shroff, " Instigation of Orthogonal Wavelet Transforms using Walsh, Cosine, Hartley, KekreTransforms and their use in Image Compression", International Journal of Computer Science and Information Security (IJCSIS), Vol 9, No. 6, pp. 125-133, 2011.

[5] H.B.Kekre, Tanuja Sarode, Sudeep Thepade, "Inception of hybrid wavelet TransformUsing Two Orthogonal Transforms and its use for Image compression", IJCSIS, vol 9, no. 6, 2011.

[6] Hong Liu, Lin Pai Zai, Ying Gao, Wen Ming Li, Jiu-Fei Zohu, "Image compressionBased on Biorthogonal Wavelet Transform", in Proc of ISCIT, 2005

[7] Loay A. George, Aree A. Mhammad, "Intra Frame Compression Using Lifting Scheme Wavelet-Based Transformation (9/7-Tap Cdf Filter)", International Conference on Multimedia Systems And Applications, MSA 2007, June 25-28, 2007, Las Vegas Nevada, USA.

[8] Arup Kumar Pal, G.P. Biswas, S. Mukhopadhyay, "A Hybrid DCT-VQ Based Approach for Efficient compression of Colour Images", International Conference on Computer and Communication Technology, (ICCCT), 2010, pp. 177-181.

[9] Adnan Khashman, and KamilDimililer, "Image Compression using Neural Networks and Haar Wavelet," WSEA Transactions on Image Processing, Vol. 4, no. 5, pp. 330- 339, 2008.

[10] G. Boopathi, Dr. S. Arockiasamy, "Image Compression: Wavelet Transform using Radial Basis function (RBF) Neural Network", IEEE Transactions, 2012, pp. 340-344. 
[11] H. B. Kekre, J. K. Solanki, "Comparative Performance of Various Trignometric Transforms For Transform Image Coding" International Journal of Electronics, Vol.44, Issue 3,pp.305- 315.

[12] H.B.Kekre, Tanuja Sarode, Prachi Natu, "Efficient Image Compression TechniqueUsing Full, Column and Row Transforms on Colour Image ", International Journal of Advances in Engineering and Technology, Vol.6, Issue 1, March 2013, pp. 88-100.

[13] H. B. Kekre, Tanuja Sarode, Prachi Natu, “ Image Compression Using Column, Row and Full Wavelet Transforms Of Walsh, Cosine, Haar, Kekre, Slant and Sine and Their Comparison With Corresponding Orthogonal Transforms", International Journal of Engineering Research and development (IJERD), Vol. 6, Issue 4, March 2013, pp.102-113. 